\title{
Recurrence Relations for Marginal and Joint Moment Generating Functions of Generalized Order Statistics from a New Class of Pareto Distributions
}

\author{
Jagdish Saran \\ Department of Statistics, University of Delhi, \\ Delhi - 110007, India \\ jagdish_saran52@yahoo.co.in \\ Kamal Nain \\ Department of Statistics, University of Delhi, \\ Delhi - 110007, India \\ kamal.180968@gmail.com
}

Received 23 November 2014

Accepted 4 April 2016

\begin{abstract}
In this paper, we establish some recurrence relations for marginal and joint moment generating functions of generalized order statistics from a new class of Pareto distributions. For a particular case these results verify the corresponding results of Athar et al. (2012).
\end{abstract}

Keywords: Generalized order statistics; single moments; product moments; marginal and joint moment generating functions; recurrence relations; Pareto distribution.

2000 Mathematics Subject Classification: 62G30, 62E10

\section{Introduction}

Generalized order statistics (GOS) have been introduced and extensively studied in Kamps (1995 a, b) as a unified theoretical set-up which contains a variety of models of ordered random variables with different interpretations. Examples of such models are: Ordinary order statistics, Sequential order statistics, Progressive type II censored order statistics, Record values, $\mathrm{k}^{\text {th }}$ record values and Pfeifer's records. There is no natural interpretation of generalized order statistics in terms of observed random samples but these models can be effectively applied in life testing and reliability analysis, medical and life time data, and models related to software reliability analysis. The common approach makes it possible to define several distributional properties at once. The structural similarities of these models are based on the similarity of their joint density function.

\section{A New Class of Pareto Distributions}

Consider a family of distributions defined by the function

$$
\mathrm{f}(\mathrm{x})=\frac{\theta \Psi^{\theta}(\alpha) \Psi_{1}(\mathrm{x})}{\Psi^{\theta+1}(\mathrm{x})}, \alpha \leq \mathrm{x}<\infty,
$$


where $\Psi(\mathrm{x})$ is some function of $\mathrm{x}$ such that $\Psi(0)=0, \Psi(\infty)=\infty$ and $\Psi_{\mathrm{n}}(0)=\left[\frac{\partial^{\mathrm{n}}(\Psi(\mathrm{x}))}{\partial \mathrm{x}^{\mathrm{n}}}\right]_{\mathrm{x}=0}<\infty$, $\mathrm{n}=1,2, \ldots$ Expanding $\Psi(\mathrm{x})$ and $\Psi_{1}(\mathrm{x})$ by using Maclaurin's theorem, we get

$$
\Psi(\mathrm{x})=\mathrm{x} \Psi_{1}(0)+\frac{\mathrm{x}^{2}}{2 !} \Psi_{2}(0)+\ldots \text { and } \Psi_{1}(\mathrm{x})=\Psi_{1}(0)+\mathrm{x} \Psi_{2}(0)+\frac{\mathrm{x}^{2}}{2 !} \Psi_{3}(0)+\ldots
$$

Case i. When $\Psi_{1}(0) \neq 0$.

After dividing the two expressions given in (2), we have

$$
\begin{aligned}
\frac{\Psi(\mathrm{x})}{\Psi_{1}(\mathrm{x})} & =\frac{\mathrm{x}\left(\Psi_{1}(0)+\frac{\mathrm{x}}{2 !} \Psi_{2}(0)+\frac{\mathrm{x}^{2}}{3 !} \Psi_{3}(0)+\ldots\right)}{\Psi_{1}(0)+\mathrm{x} \Psi_{2}(0)+\frac{\mathrm{x}^{2}}{2 !} \Psi_{3}(0)+\ldots} \\
& =\frac{\mathrm{x}\left(1+\mathrm{a}_{1} \mathrm{x}+\mathrm{a}_{2} \mathrm{x}^{2}+\ldots\right)}{1+2 \mathrm{a}_{1} \mathrm{x}+3 \mathrm{a}_{2} \mathrm{x}^{2}+\ldots}, \text { where } \mathrm{a}_{\mathrm{n}}=\frac{\Psi_{\mathrm{n}+1}(0)}{(\mathrm{n}+1) ! \Psi_{1}(0)}, \mathrm{n}=1,2, \ldots \\
& =\mathrm{x}\left(1+\mathrm{a}_{1} \mathrm{x}+\mathrm{a}_{2} \mathrm{x}^{2}+\ldots\right)\left(\sum_{\delta=0}^{\infty}(-1)^{\delta}\left(2 \mathrm{a}_{1} \mathrm{x}+3 \mathrm{a}_{2} \mathrm{x}^{2}+\ldots\right)^{\delta}\right) \\
& =\mathrm{x}+\sum_{\delta=2}^{\infty} \mathrm{b}_{\delta} \mathrm{x}^{\delta},
\end{aligned}
$$

where $b_{\delta}$ is the coefficient of $x^{\delta}$ in the product

$$
\left(1+\mathrm{a}_{1} \mathrm{x}+\mathrm{a}_{2} \mathrm{x}^{2}+\ldots\right)\left(\sum_{\delta=0}^{\infty}(-1)^{\delta}\left(2 \mathrm{a}_{1} \mathrm{x}+3 \mathrm{a}_{2} \mathrm{x}^{2}+\ldots\right)^{\delta}\right)
$$

The procedure for computing $b_{\delta}$ will be explained in the illustrations considered in Section 5 .

Case ii. When $\Psi_{s}(0)=0, s=1,2,3, \ldots, p-1$ and $\Psi_{p}(0) \neq 0$. In this case,

$$
\begin{aligned}
\frac{\Psi(x)}{\Psi_{1}(x)}= & \frac{\frac{x}{p}\left(\Psi_{p}(0)+\frac{x}{(p+1)} \Psi_{p+1}(0)+\frac{x^{2}}{(p+1)(p+2)} \Psi_{p+2}(0)+\ldots\right)}{\Psi_{p}(0)+\frac{x}{p} \Psi_{p+1}(0)+\ldots} \\
& =\frac{x}{p}+\sum_{\delta=2}^{\infty} b_{\delta}^{\prime} x^{\delta}
\end{aligned}
$$

(obtained by repeating the similar steps). 
The cumulative distribution function $(c d f)$ and the probability density function $(p d f)$ of the random variable $\mathrm{X}$ are connected by the relation,

$$
1-F(x)=\frac{\Psi(x)}{\theta \Psi_{1}(x)} f(x)= \begin{cases}\frac{1}{\theta}\left(x+\sum_{\delta=2}^{\infty} b_{\delta} x^{\delta}\right) f(x), & \text { under } A \\ \frac{1}{\theta}\left(\frac{x}{p}+\sum_{\delta=2}^{\infty} b_{\delta}^{\prime} x^{\delta}\right) f(x), & \text { under } B\end{cases}
$$

where $\mathrm{A} \equiv\left\{\Psi_{1}(0) \neq 0\right\}$ and $\mathrm{B} \equiv\left\{\Psi_{\mathrm{s}}(0)=0, \mathrm{~s}=1,2,3, \ldots, \mathrm{p}-1\right.$ and $\left.\Psi_{\mathrm{p}}(0) \neq 0\right\}$.

The mathematical form of the cdf, as given in (5), plays an important role for deriving the recurrence relations for single and product moments of GOS from the new class of Pareto distributions (1).

\section{Generalized Order Statistics}

Let $\left\{X_{n}, n \geq 1\right\}$ be a sequence of absolutely continuous, independent and identically distributed random variables with cdf $\mathrm{F}(\mathrm{x})=\mathrm{P}(\mathrm{X} \leq \mathrm{x})$ and $\mathrm{pdf} \mathrm{f}(\mathrm{x})$. Assume $\mathrm{k}>0, \mathrm{n} \in\{2,3, \ldots\}$, $\tilde{\mathrm{m}}=\left(\mathrm{m}_{1}, \mathrm{~m}_{2}, \ldots, \mathrm{m}_{\mathrm{n}-1}\right) \in \mathrm{R}^{\mathrm{n}-1}, \mathrm{M}_{\mathrm{r}}=\sum_{\mathrm{j}=\mathrm{r}}^{\mathrm{n}-1} \mathrm{~m}_{\mathrm{j}}$, such that $\gamma_{\mathrm{r}}=\mathrm{k}+\mathrm{n}-\mathrm{r}+\mathrm{M}_{\mathrm{r}}>0$ for all $\mathrm{r} \in\{1,2, \ldots, \mathrm{n}-1\}$. Then $X(r, n, \tilde{m}, k), r=1,2, \ldots, n$, are called GOS if their joint pdf is given by

$$
\begin{aligned}
\mathrm{f}^{\mathrm{X}(1, \mathrm{n}, \tilde{\mathrm{m}}, \mathrm{k}), \mathrm{x}(2, \mathrm{n}, \tilde{\mathrm{m}}, \mathrm{k}), \ldots, \mathrm{X}(\mathrm{n}, \mathrm{n}, \tilde{\mathrm{m}}, \mathrm{k})}\left(\mathrm{x}_{1}, \mathrm{x}_{2}, \ldots, \mathrm{x}_{\mathrm{n}}\right)=\mathrm{k}\left(\prod_{\mathrm{j}=1}^{\mathrm{n}-1} \gamma_{\mathrm{j}}\right)\left(\prod_{\mathrm{i}=1}^{\mathrm{n}-1}\left(1-\mathrm{F}\left(\mathrm{x}_{\mathrm{i}}\right)\right)^{\mathrm{m}_{\mathrm{i}}} \mathrm{f}\left(\mathrm{x}_{\mathrm{i}}\right)\right) \\
\\
\times\left(1-\mathrm{F}\left(\mathrm{x}_{\mathrm{n}}\right)\right)^{\mathrm{k}-1} \mathrm{f}\left(\mathrm{x}_{\mathrm{n}}\right),
\end{aligned}
$$

where $\mathrm{F}^{-1}(0+)<\mathrm{x}_{1} \leq \mathrm{x}_{2} \leq \ldots \leq \mathrm{x}_{\mathrm{n}}<\mathrm{F}^{-1}(1)$.

By choosing appropriate values of parameters, we get the distribution of a few very common statistics as shown in Table 1, given below.

Table 1

\begin{tabular}{lll}
\hline S. No. & Choice of parameters for $\mathrm{i}=1,2, \ldots, \mathrm{n}$ & GOS become \\
\hline 1 & $\begin{array}{l}\gamma_{\mathrm{i}}=\mathrm{n}-\mathrm{i}+1, \mathrm{~m}_{1}=\mathrm{m}_{2}=\ldots=\mathrm{m}_{\mathrm{n}-1}=0 \text { and } \\
\mathrm{k}=1\end{array}$ & Ordinary order statistics \\
2 & $\gamma_{\mathrm{i}}=\mathrm{k}, \mathrm{m}_{1}=\mathrm{m}_{2}=\ldots=\mathrm{m}_{\mathrm{n}-1}=-1, \mathrm{k} \in \mathrm{N}$ & $\mathrm{k}^{\text {th }}$ record values \\
3 & $\gamma_{\mathrm{i}}=(\mathrm{n}-\mathrm{i}+1) \alpha_{\mathrm{i}}, \alpha_{\mathrm{i}}>0 \square$ & Sequential order statistics \\
4 & $\gamma_{\mathrm{i}}=\alpha-\mathrm{i}+1, \alpha>0$ & Order statistics with non integer sample size \\
5 & $\gamma_{\mathrm{i}}=\beta_{\mathrm{i}}, \beta_{\mathrm{i}}>0$ & Pfeifer's record values \\
6 & $\mathrm{~m}_{\mathrm{i}} \in \mathrm{N}_{\mathrm{o}}, \mathrm{k} \in \mathrm{N}$ & $\begin{array}{l}\text { Progressively type-II right censored order } \\
\text { statistics }\end{array}$ \\
\hline
\end{tabular}


The joint pdf of first $r$ GOS is given by:

$$
\begin{aligned}
f^{X(1, n, \tilde{m}, k), x(2, n, \tilde{m}, k), \ldots, x(r, n, \tilde{m}, k)}\left(x_{1}, x_{2}, \ldots, x_{r}\right)=c_{r-1}( & \left.\prod_{i=1}^{r-1}\left(1-F\left(x_{i}\right)\right)^{m_{i}} f\left(x_{i}\right)\right) \\
& \times\left(1-F\left(x_{r}\right)\right)^{k+n-r+M_{r}-1} f\left(x_{r}\right),
\end{aligned}
$$

where $\mathrm{F}^{-1}(0+)<\mathrm{x}_{1} \leq \mathrm{x}_{2} \leq \ldots \leq \mathrm{x}_{\mathrm{r}}<\mathrm{F}^{-1}(1)$.

We now consider two cases:

Case I: $\mathrm{m}_{1}=\mathrm{m}_{2}=\ldots=\mathrm{m}_{\mathrm{n}-1}=\mathrm{m}$

Case II: $\gamma_{\mathrm{i}} \neq \boldsymbol{\gamma}_{\mathrm{j}} ; \mathrm{i} \neq \mathrm{j}, \quad \mathrm{i}, \mathrm{j}=1,2, \ldots, \mathrm{n}-1$.

For case I, the GOS will be denoted by X(r, n, m, k). The pdf of X(r, n, m, k) is given by

$$
\mathrm{f}^{\mathrm{x}(\mathrm{r}, \mathrm{n}, \mathrm{m}, \mathrm{k})}(\mathrm{x})=\frac{\mathrm{c}_{\mathrm{r}-1}}{(\mathrm{r}-1) !}(1-\mathrm{F}(\mathrm{x}))^{\gamma_{\mathrm{r}}-1} \mathrm{f}(\mathrm{x}) \mathrm{g}_{\mathrm{m}}^{\mathrm{r}-1}(\mathrm{~F}(\mathrm{x})), \quad \mathrm{x} \in \mathrm{R},
$$

and the joint pdf of $X(r, n, m, k)$ and $X(s, n, m, k), \quad 1 \leq r<s \leq n$, is given by :

$$
\begin{aligned}
& \mathrm{f}^{\mathrm{X}(\mathrm{r}, \mathrm{n}, \mathrm{m}, \mathrm{k}), \mathrm{x}(\mathrm{s}, \mathrm{n}, \mathrm{m}, \mathrm{k})}(\mathrm{x}, \mathrm{y})=\frac{\mathrm{c}_{\mathrm{s}-1}}{(\mathrm{r}-1) !(\mathrm{s}-\mathrm{r}-1) !}\left((1-\mathrm{F}(\mathrm{x}))^{\mathrm{m}} \mathrm{f}(\mathrm{x})\right) \mathrm{g}_{\mathrm{m}}^{\mathrm{r}-1}(\mathrm{~F}(\mathrm{x})) \\
& \times\left[\mathrm{h}_{\mathrm{m}}(\mathrm{F}(\mathrm{y}))-\mathrm{h}_{\mathrm{m}}(\mathrm{F}(\mathrm{x}))\right]^{\mathrm{s}-\mathrm{r}-1} \\
& \times(1-\mathrm{F}(\mathrm{y}))^{\gamma_{\mathrm{s}}-1} \mathrm{f}(\mathrm{y}), \quad-\infty<\mathrm{x}<\mathrm{y}<\infty \text {, }
\end{aligned}
$$

where

$$
\begin{aligned}
& \mathrm{c}_{\mathrm{r}-1}=\prod_{\mathrm{j}=1}^{\mathrm{r}} \gamma_{\mathrm{j}}, \quad \gamma_{\mathrm{j}}=\mathrm{k}+(\mathrm{n}-\mathrm{j})(\mathrm{m}+1), \mathrm{r}=1,2, \ldots, \mathrm{n}, \\
& \mathrm{g}_{\mathrm{m}}(\mathrm{x})=\mathrm{h}_{\mathrm{m}}(\mathrm{x})-\mathrm{h}_{\mathrm{m}}(0), \mathrm{x} \in(0,1) \text { and } \\
& \mathrm{h}_{\mathrm{m}}(\mathrm{x})= \begin{cases}-\frac{(1-\mathrm{x})^{\mathrm{m}+1}}{\mathrm{~m}+1}, & \mathrm{~m} \neq-1, \\
-\log (1-\mathrm{x}), & \mathrm{m}=-1 .\end{cases}
\end{aligned}
$$

For case II, the GOS will be denoted by $X(r, n, \tilde{m}, k)$. The pdf of $X(r, n, \tilde{m}, k)$ is given by

$$
\mathrm{f}^{\mathrm{X}(\mathrm{r}, \mathrm{n}, \tilde{\mathrm{m}}, \mathrm{k})}(\mathrm{x})=\mathrm{c}_{\mathrm{r}-1} \mathrm{f}(\mathrm{x}) \sum_{\mathrm{i}=1}^{\mathrm{r}} \mathrm{a}_{\mathrm{i}}(\mathrm{r})(1-\mathrm{F}(\mathrm{x}))^{\gamma_{\mathrm{i}}-1}, \quad \mathrm{x} \in \mathrm{R},
$$

and the joint pdf of $X(r, n, \tilde{m}, k)$ and $X(s, n, \tilde{m}, k), 1 \leq r<s \leq n$, is given by

$$
\begin{aligned}
& \mathrm{f}^{\mathrm{X}(\mathrm{r}, \mathrm{n}, \tilde{\mathrm{m}}, \mathrm{k}), \mathrm{X}(\mathrm{s}, \mathrm{n}, \tilde{\mathrm{m}}, \mathrm{k})}(\mathrm{x}, \mathrm{y})=\mathrm{c}_{\mathrm{s}-1}\left\{\sum_{\mathrm{i}=\mathrm{r}+1}^{\mathrm{s}} \mathrm{a}_{\mathrm{i}}^{\mathrm{r}}(\mathrm{s})\left(\frac{1-\mathrm{F}(\mathrm{y})}{1-\mathrm{F}(\mathrm{x})}\right)^{\gamma_{\mathrm{i}}}\right\}\left\{\sum_{\mathrm{i}=1}^{\mathrm{r}} \mathrm{a}_{\mathrm{i}}(\mathrm{r})(1-\mathrm{F}(\mathrm{x}))^{\gamma_{\mathrm{i}}}\right\} \\
& \times \frac{\mathrm{f}(\mathrm{x})}{1-\mathrm{F}(\mathrm{x})} \frac{\mathrm{f}(\mathrm{y})}{1-\mathrm{F}(\mathrm{y})}, \quad-\infty<\mathrm{x}<\mathrm{y}<\infty,
\end{aligned}
$$

where $c_{s-1}=\prod_{j=1}^{s} \gamma_{j}, \quad \gamma_{j}=k+n-j+M_{j}, s=1,2, \ldots, n$. 
Further, it can be proved that

$$
\begin{aligned}
& a_{i}(r)=\prod_{j(\neq i)=1}^{r}\left(\gamma_{j}-\gamma_{i}\right)^{-1}, 1 \leq i \leq r \leq n \\
& a_{i}^{r}(s)=\prod_{j(\neq i)=r+1}^{s}\left(\gamma_{j}-\gamma_{i}\right)^{-1}, r+1 \leq i \leq s \leq n \\
& a_{i}(r)=\left(\gamma_{r+1}-\gamma_{i}\right) a_{i}(r+1) \\
& c_{r}=c_{r-1} \gamma_{r+1} \\
& \sum_{i=1}^{r} a_{i}(r)=0 \\
& \sum_{i=r+1}^{s} a_{i}^{r}(s)=0 .
\end{aligned}
$$

The moments of order statistics have generated considerable interest in the recent years. Several recurrence relations and identities satisfied by single as well as product moments of order statistics have been obtained by various authors in the past. These relations help in reducing the quantum of computations involved. Joshi $(1978,1982)$ established recurrence relations for exponential distribution with unit mean and were further extended by Balakrishnan and Joshi (1984) for doubly truncated exponential distribution. For linear exponential distribution, Balakrishnan and Malik(1986) derived the similar type of relations which were extended to doubly truncated linear exponential distribution by Mohie El-Din et al. (1997) and Saran and Pushkarna (1999). Nain (2010 a, b) obtained recurrence relations for moments of ordinary order statistics and those of $\mathrm{k}^{\text {th }}$ record values from $\mathrm{p}^{\text {th }}$ order exponential and generalized Weibull distributions, respectively.

The recurrence relations for the moments of generalized order statistics based on non identically distributed random variables were developed by Kamps (1995 a, b). Pawlas and Szynal (2001) obtained recurrence relations for single and product moments of generalized order statistics from Pareto, generalized Pareto and Burr distributions. Saran and Pandey $(2004,2009)$ obtained recurrence relations for single and product moments of generalized order statistics from linear exponential and Burr distributions. Saran and Pandey (2011) obtained recurrence relations for marginal and joint moment generating functions of dual (lower) generalized order statistics from inverse Weibull distribution. The other similar work also appear in Saran and Nain (2012 a, b, c).

In this paper, we have established recurrence relations for marginal and joint moment generating functions (and hence for the single and product moments) of GOS from a new class of Pareto distributions. This distribution has many applications in Economics and models related to income of an individual or of a firm. The results so obtained are generalized versions of some of the recurrence relations obtained by Ather et al. (2012).

\section{Review of a few identities of Athar and Islam (2004)}

Here we write a few identities, stated in the form of Lemmas 2.1, 2.3, 3.1 and 3.2, respectively, in Athar and Islam (2004) for Borel measurable functions $\omega(\mathrm{x})$ and $\omega(\mathrm{x}, \mathrm{y})$ with support $(\alpha, \infty)$.

$$
\begin{aligned}
& E(\omega(X(r, n, m, k)))-E(\omega(X(r-1, n, m, k))) \\
& =\frac{c_{r-2}}{(r-1) !} \int_{\alpha}^{\infty} \frac{\partial \omega(x)}{\partial x}(\bar{F}(x))^{\gamma_{r}} f(x) g_{m}^{r-1}(F(x)) d x
\end{aligned}
$$


(ii) $\quad \mathrm{E}(\omega(\mathrm{X}(\mathrm{r}, \mathrm{n}, \tilde{\mathrm{m}}, \mathrm{k})))-\mathrm{E}(\omega(\mathrm{X}(\mathrm{r}-1, \mathrm{n}, \tilde{\mathrm{m}}, \mathrm{k})))$

$$
=\frac{\mathrm{c}_{\mathrm{r}-1}}{\gamma_{\mathrm{r}}} \int_{\alpha}^{\infty} \frac{\partial \omega(\mathrm{x})}{\partial \mathrm{x}}\left[\sum_{\mathrm{i}=1}^{\mathrm{r}} \mathrm{a}_{\mathrm{i}}(\mathrm{r})(1-\mathrm{F}(\mathrm{x}))^{\gamma_{\mathrm{i}}}\right] \mathrm{dx}
$$

(iii) $\quad E(\omega(X(r, n, m, k), X(s, n, m, k)))-E(\omega(X(r, n, m, k), X(s-1, n, m, k)))$

$$
\begin{aligned}
& =\frac{\mathrm{c}_{\mathrm{s}-2}}{(\mathrm{r}-1) !(\mathrm{s}-\mathrm{r}-1) !} \int_{\alpha}^{\infty} \int_{\mathrm{x}}^{\infty} \frac{\partial \omega(\mathrm{x}, \mathrm{y})}{\partial \mathrm{y}}(\overline{\mathrm{F}}(\mathrm{x}))^{\mathrm{m}} \mathrm{f}(\mathrm{x}) \mathrm{g}_{\mathrm{m}}^{\mathrm{r}-1}(\mathrm{~F}(\mathrm{x})) \\
& \quad \times\left[\mathrm{h}_{\mathrm{m}}(\mathrm{F}(\mathrm{y}))-\mathrm{h}_{\mathrm{m}}(\mathrm{F}(\mathrm{x}))\right]^{\mathrm{s}-\mathrm{r}-1}(\overline{\mathrm{F}}(\mathrm{y}))^{\gamma_{\mathrm{s}}} \mathrm{dydx}
\end{aligned}
$$

(iv) $\quad E(\omega(X(r, n, \tilde{m}, k), X(s, n, \tilde{m}, k)))-E(\omega(X(r, n, \tilde{m}, k), X(s-1, n, \tilde{m}, k)))$

$$
=c_{s-2} \int_{\alpha}^{\infty} \int_{x}^{\infty} \frac{\partial \omega(x, y)}{\partial y}\left[\sum_{i=1}^{r} a_{i}(r)(\bar{F}(x))^{\gamma_{i}}\right] \frac{f(x)}{\bar{F}(x)} \cdot\left[\sum_{i=r+1}^{s} a_{i}^{r}(s)\left(\frac{\bar{F}(y)}{\bar{F}(x)}\right)^{\gamma_{i}}\right] d y d x,
$$

where $\bar{F}(x)=1-F(x)$.

\section{Notations}

The following symbols and notations will be used in the paper:

(i) $\mathrm{A} \equiv\left\{\Psi_{1}(0) \neq 0\right\}$

(ii) $\mathrm{B} \equiv\left\{\Psi_{\mathrm{s}}(0)=0, \mathrm{~s}=1,2,3, \ldots, \mathrm{p}-1\right.$ and $\left.\Psi_{\mathrm{p}}(0) \neq 0\right\}$

For $\mathrm{n}=1,2,3, \ldots, 1 \leq \mathrm{r}<\mathrm{s} \leq \mathrm{n}, \mathrm{k} \geq 1$ and $\mathrm{u}, \mathrm{v} \in\{0,1,2, \ldots\}$, we denote by
(iii) $\quad(\mathrm{n})_{\mathrm{r}}=\mathrm{n}(\mathrm{n}-1)(\mathrm{n}-2) \ldots(\mathrm{n}-\mathrm{r}+1)$
(iv) $\quad \mu_{\mathrm{r}: \mathrm{n}, \mathrm{m}, \mathrm{k}}^{\mathrm{u}}=\mathrm{E}(\mathrm{X}(\mathrm{r}, \mathrm{n}, \mathrm{m}, \mathrm{k}))^{\mathrm{u}}$
(v) $\quad \mu_{\mathrm{r}, \mathrm{s}: \mathrm{n}, \mathrm{m}, \mathrm{k}}^{\mathrm{u}, \mathrm{r}}=\mathrm{E}\left[\{\mathrm{X}(\mathrm{r}, \mathrm{n}, \mathrm{m}, \mathrm{k})\}^{\mathrm{u}}\{\mathrm{X}(\mathrm{s}, \mathrm{n}, \mathrm{m}, \mathrm{k})\}^{\mathrm{v}}\right]$
(vi) $\quad \mu_{\mathrm{r}: \mathrm{n}, \tilde{\mathrm{m}}, \mathrm{k}}^{\mathrm{u}}=\mathrm{E}(\mathrm{X}(\mathrm{r}, \mathrm{n}, \tilde{\mathrm{m}}, \mathrm{k}))^{\mathrm{u}}$
(vii) $\quad \mu_{\mathrm{r}, \mathrm{s}: \mathrm{n}, \tilde{\mathrm{m}}, \mathrm{k}}^{\mathrm{u}, \mathrm{m}}=\mathrm{E}\left[\{\mathrm{X}(\mathrm{r}, \mathrm{n}, \tilde{\mathrm{m}}, \mathrm{k})\}^{\mathrm{u}}\{\mathrm{X}(\mathrm{s}, \mathrm{n}, \tilde{\mathrm{m}}, \mathrm{k})\}^{\mathrm{v}}\right]$
(viii) $\quad \mathrm{M}_{\mathrm{r}: \mathrm{n}, \mathrm{m}, \mathrm{k}}(\mathrm{t})=\mathrm{E}\left(\mathrm{e}^{\mathrm{tX}(\mathrm{r}, \mathrm{n}, \mathrm{m}, \mathrm{k})}\right)$ and $\mathrm{M}_{\mathrm{r}: \mathrm{n}, \tilde{\mathrm{m}}, \mathrm{k}}(\mathrm{t})=\mathrm{E}\left(\mathrm{e}^{\mathrm{tX}(\mathrm{r}, \mathrm{n}, \tilde{\mathrm{m}}, \mathrm{k})}\right)$
(ix) $\quad \mathrm{M}_{\mathrm{r}: \mathrm{n}, \mathrm{m}, \mathrm{k}}^{\mathrm{u}}(\mathrm{t})=\frac{\partial^{\mathrm{u}}}{\partial \mathrm{t}^{\mathrm{u}}}\left[\mathrm{M}_{\mathrm{r}: \mathrm{n}, \mathrm{m}, \mathrm{k}}(\mathrm{t})\right]$ and $\mathrm{M}_{\mathrm{r}: \mathrm{n}, \mathrm{m}, \mathrm{k}}^{\mathrm{u}}(0)=\mu_{\mathrm{r}: \mathrm{n}, \mathrm{m}, \mathrm{k}}^{\mathrm{u}}$
(x) $\quad M_{r: n, \tilde{m}, k}^{u}(t)=\frac{\partial^{u}}{\partial t^{u}}\left[M_{r: n, \tilde{m}, k}(t)\right]$ and $M_{r: n, \tilde{m}, k}^{u}(0)=\mu_{r: n, \tilde{m}, k}^{u}$

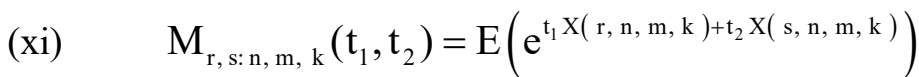
(xii) $\quad \mathrm{M}_{\mathrm{r}, \mathrm{s}: \mathrm{n}, \tilde{\mathrm{m}}, \mathrm{k}}\left(\mathrm{t}_{1}, \mathrm{t}_{2}\right)=\mathrm{E}\left(\mathrm{e}^{\mathrm{t}_{1} \mathrm{X}(\mathrm{r}, \mathrm{n}, \tilde{\mathrm{m}}, \mathrm{k})+\mathrm{t}_{2} \mathrm{X}(\mathrm{s}, \mathrm{n}, \tilde{\mathrm{m}}, \mathrm{k})}\right)$ 
(xiii)

$$
\begin{aligned}
& M_{r, s: n, m, k}^{u, v}\left(t_{1}, t_{2}\right)=\frac{\partial^{u+v}}{\partial t_{1}^{u} \partial t_{2}^{v}}\left[M_{r, s: n, m, k}\left(t_{1}, t_{2}\right)\right] \text { and } M_{r, s: n, m, k}^{u, v}(0,0)=\mu_{r, s: n, m, k}^{u, v} \\
& M_{r, s: n, \tilde{m}, k}^{u, v}\left(t_{1}, t_{2}\right)=\frac{\partial^{u+v}}{\partial t_{1}^{u} \partial t_{2}^{v}}\left[M_{r, s: n, \tilde{m}, k}\left(t_{1}, t_{2}\right)\right] \text { and } M_{r, s: n, \tilde{m}, k}^{u, v}(0,0)=\mu_{r, s: n, \tilde{m}, k}^{u, v}
\end{aligned}
$$

\section{Recurrence Relations for Moment Generating Functions}

In this section, we shall derive recurrence relations for marginal and joint moment generating functions of generalized order statistics from the new class of Pareto distributions given in (1).

Case I: $\mathrm{m}_{1}=\mathrm{m}_{2}=\ldots=\mathrm{m}_{\mathrm{n}-1}=\mathrm{m}$.

Theorem 1. Fix a positive integer $k$. For $n \in N, m \in Z$ and $1 \leq r \leq n$,

$$
M_{r: n, m, k}(t)-M_{r-1: n, m, k}(t)=\left\{\begin{array}{l}
\frac{t}{\theta \gamma_{r}}\left(M_{r: n, m, k}^{1}(t)+\sum_{\delta=2}^{\infty} b_{\delta} M_{r: n, m, k}^{\delta}(t)\right), \text { under A, } \\
\frac{t}{\theta \gamma_{r}}\left(\frac{M_{r: n, m, k}^{1}(t)}{p}+\sum_{\delta=2}^{\infty} b_{\delta}^{\prime} M_{r: n, m, k}^{\delta}(t)\right), \text { under B. }
\end{array}\right.
$$

Proof. Using (8), the moment generating function of $\mathrm{X}(\mathrm{r}, \mathrm{n}, \mathrm{m}, \mathrm{k})$ from the new class of Pareto distributions (1) is given by

$$
M_{r: n, m, k}(t)=\frac{c_{r-1}}{(r-1) !} \int_{\alpha}^{\infty} e^{t x}(\bar{F}(x))^{\gamma_{r}-1} f(x) g_{m}^{r-1}(F(x)) d x .
$$

Substituting $\omega(\mathrm{x})=\mathrm{e}^{\mathrm{tx}}$ in (13) and using (5) for case A, we have

$$
\begin{aligned}
M_{r: n, m, k}(t)-M_{r-1: n, m, k}(t)= & \frac{t c_{r-1}}{\theta \gamma_{r}(r-1) !} \int_{\alpha}^{\infty} e^{t x}(\bar{F}(x))^{\gamma_{r}-1} \\
& \times\left(x+\sum_{\delta=2}^{\infty} b_{\delta} x^{\delta}\right) f(x) g_{m}^{r-1}(F(x)) d x,
\end{aligned}
$$

which after simplification leads to case A of (17). Likewise, the case B of (17) can be easily established.

Remark 1. After differentiating both sides of (17), with respect to $t, u$ times and putting $t=0$, we shall derive the recurrence relation among moments of generalized order statistics from the new class of Pareto distributions given in (1) as: 


$$
\mu_{\mathrm{r}: \mathrm{n}, \mathrm{m}, \mathrm{k}}^{\mathrm{u}}= \begin{cases}\frac{1}{\theta \gamma_{\mathrm{r}}-\mathrm{u}}\left(\theta \gamma_{\mathrm{r}} \mu_{\mathrm{r}-1: \mathrm{n}, \mathrm{m}, \mathrm{k}}^{\mathrm{u}}+\mathrm{u} \sum_{\delta=2}^{\infty} \mathrm{b}_{\delta} \mu_{\mathrm{r}: \mathrm{n}, \mathrm{m}, \mathrm{k}}^{\mathrm{u}+\delta-1}\right), & \text { under A, } \\ \frac{\mathrm{p}}{\mathrm{p} \theta \gamma_{\mathrm{r}}-\mathrm{u}}\left(\theta \gamma_{\mathrm{r}} \mu_{\mathrm{r}-1: \mathrm{n}, \mathrm{m}, \mathrm{k}}^{\mathrm{u}}+\mathrm{u} \sum_{\delta=2}^{\infty} \mathrm{b}_{\delta}^{\prime} \mu_{\mathrm{r}: \mathrm{n}, \mathrm{m}, \mathrm{k}}^{\mathrm{u}+\delta-1}\right), & \text { under B. }\end{cases}
$$

Theorem 2. Fix a positive integer $k$. For $n \in N, m \in Z$ and $1 \leq r<s \leq n$,

$$
\begin{aligned}
& M_{r, s: n, m, k}\left(t_{1}, t_{2}\right)-M_{r, s-1: n, m, k}\left(t_{1}, t_{2}\right) \\
& =\left\{\begin{array}{l}
\frac{t_{2}}{\theta \gamma_{s}}\left(M_{r, s: n, m, k}^{0,1}\left(t_{1}, t_{2}\right)+\sum_{\delta=2}^{\infty} b_{\delta} M_{r, s: n, m, k}^{0, \delta}\left(t_{1}, t_{2}\right)\right), \text { under } A, \\
\frac{t_{2}}{\theta \gamma_{s}}\left(\frac{M_{r, s: n, m, k}^{0,1}\left(t_{1}, t_{2}\right)}{p}+\sum_{\delta=2}^{\infty} b_{\delta}^{\prime} M_{r, s: n, m, k}^{0, \delta}\left(t_{1}, t_{2}\right)\right), \text { under B. }
\end{array}\right.
\end{aligned}
$$

Proof. Using (9), the expression for joint moment generating function of $\mathrm{X}(\mathrm{r}, \mathrm{n}, \mathrm{m}, \mathrm{k})$ and $\mathrm{X}(\mathrm{s}, \mathrm{n}, \mathrm{m}, \mathrm{k})$ is:

$$
\begin{aligned}
M_{r, s: n, m, k}\left(t_{1}, t_{2}\right)=\frac{c_{s-1}}{(r-1) !(s-r-1) !} \int_{\alpha}^{\infty} \int_{x}^{\infty} e^{t_{1} x+t_{2} y}(\bar{F}(x))^{m} f(x) g_{m}^{r-1}(F(x)) \\
\times\left[h_{m}(F(y))-h_{m}(F(x))\right]^{s-r-1}(\bar{F}(y))^{\gamma_{s}-1} f(y) d y d x .
\end{aligned}
$$

Substituting $\omega(\mathrm{x}, \mathrm{y})=\mathrm{e}^{\mathrm{t}_{1} \mathrm{x}+\mathrm{t}_{2} \mathrm{y}}$ in (15) and using (5) for case A, we have

$$
\begin{aligned}
& M_{r, s: n, m, k}\left(t_{1}, t_{2}\right)-M_{r, s-1: n, m, k}\left(t_{1}, t_{2}\right)=\frac{t_{2} c_{s-2}}{(r-1) !(s-r-1) !} \int_{\alpha}^{\infty} \int_{x}^{\infty} e^{t_{1} x+t_{2} y}(\bar{F}(x))^{m} f(x) g_{m}^{r-1}(F(x)) \\
& \times\left[h_{m}(F(y))-h_{m}(F(x))\right]^{s-r-1}(\bar{F}(y))^{\gamma_{s}} d y d x \\
&=\frac{t_{2} c_{s-1}}{\theta \gamma_{s}(r-1) !(s-r-1) !} \int_{\alpha}^{\infty} \int_{x}^{\infty} e^{t_{1} x+t_{2} y}\left(y+\sum_{\delta=2}^{\infty} b_{\delta} y^{\delta}\right)(\bar{F}(x))^{m} f(x) g_{m}^{r-1}(F(x)) \\
& \times\left[h_{m}(F(y))-h_{m}(F(x))\right]^{s-r-1}(\bar{F}(y))^{\gamma_{s}-1} f(y) d y d x
\end{aligned}
$$

which on further simplification takes the form as stated in (21) under case A. By following similar lines, the case B of (21) can easily be established.

Remark 2. After differentiating both sides of (21), with respect to $t_{1}$, $u$ times and with respect to $t_{2}, v$ times and then putting $t_{1}=t_{2}=0$, we shall derive the recurrence relation for product moments of generalized order statistics as: 


$$
\mu_{\mathrm{r}, \mathrm{s}: \mathrm{n}, \mathrm{m}, \mathrm{k}}^{\mathrm{u}, \mathrm{s}}=\left\{\begin{array}{l}
\frac{1}{\theta \gamma_{\mathrm{s}}-\mathrm{v}}\left(\theta \gamma_{\mathrm{s}} \mu_{\mathrm{r}, \mathrm{s}-1: \mathrm{n}, \mathrm{m}, \mathrm{k}}^{\mathrm{u}, \mathrm{v}}+\mathrm{v} \sum_{\delta=2}^{\infty} \mathrm{b}_{\delta} \mu_{\mathrm{r}, \mathrm{s}: \mathrm{n}, \mathrm{m}, \mathrm{k}}^{\mathrm{u}, \mathrm{v}+\delta-1}\right), \text { under } \mathrm{A}, \\
\frac{\mathrm{p}}{\mathrm{p} \theta \gamma_{\mathrm{s}}-\mathrm{v}}\left(\theta \gamma_{\mathrm{s}} \mu_{\mathrm{r}, \mathrm{s}-1: \mathrm{n}, \mathrm{m}, \mathrm{k}}^{\mathrm{u}, \mathrm{v}}+\mathrm{v} \sum_{\delta=2}^{\infty} \mathrm{b}_{\delta}^{\prime} \mu_{\mathrm{r}, \mathrm{s}: \mathrm{n}, \mathrm{m}, \mathrm{k}}^{\mathrm{u}, \mathrm{v}+\delta 1}\right), \text { under B. }
\end{array}\right.
$$

Case II: $\boldsymbol{\gamma}_{\mathrm{i}} \neq \boldsymbol{\gamma}_{\mathrm{j}} ; \mathrm{i} \neq \mathrm{j}, \quad \mathrm{i}, \mathrm{j}=1,2, \ldots, \mathrm{n}-1$.

Theorem 3. Fix a positive integer $k$. For $n \in N, m \in Z$ and $1 \leq r \leq n$,

$$
M_{r: n, \tilde{m}, k}(t)-M_{r-1: n, \tilde{m}, k}(t)=\left\{\begin{array}{l}
\frac{t}{\theta \gamma_{r}}\left(M_{r: n, \tilde{m}, k}^{1}(t)+\sum_{\delta=2}^{\infty} b_{\delta} M_{r: n, \tilde{m}, k}^{\delta}(t)\right), \text { under A, } \\
\frac{t}{\theta \gamma_{r}}\left(\frac{M_{r: n, \tilde{m}, k}^{1}(t)}{p}+\sum_{\delta=2}^{\infty} b_{\delta}^{\prime} M_{r: n, \tilde{m}, k}^{\delta}(t)\right), \text { under B. }
\end{array}\right.
$$

Proof. On using (11), the moment generating function of $X(r, n, \tilde{m}, k)$ is given by

$$
M_{r: n, \tilde{m}, k}(t)=c_{r-1} \int_{\alpha}^{\infty} e^{t x} \sum_{i=1}^{r} a_{i}(r)(1-F(x))^{\gamma_{i}-1} f(x) d x .
$$

Substituting $\omega(\mathrm{x})=\mathrm{e}^{\mathrm{tx}}$ in (14) and from (5) for case A, we have

$$
\begin{aligned}
M_{r: n, \tilde{m}, k}(t)-M_{r-1: n, \tilde{m}, k}(t)=\frac{t c_{r-1}}{\theta \gamma_{r}} \int_{\alpha}^{\infty} e^{t x}\left[\sum_{i=1}^{r} a_{i}(r)(1-F(x))^{\gamma_{i}-1}\right] & \\
& \times\left[x+\sum_{\delta=2}^{\infty} b_{\delta} x^{\delta}\right] f(x) d x
\end{aligned}
$$

which on further simplification gives (26) for case A. The case B of (26) can similarly be established.

Remark 3. After differentiating both sides of (26), with respect to $t$, $u$ times and then putting $t=0$, we shall derive the recurrence relation for moments as

$$
\mu_{\mathrm{r}: \mathrm{n}, \tilde{\mathrm{m}}, \mathrm{k}}^{\mathrm{u}}=\left\{\begin{array}{l}
\frac{1}{\theta \gamma_{\mathrm{r}}-\mathrm{u}}\left(\theta \gamma_{\mathrm{r}} \mu_{\mathrm{r}-1: \mathrm{n}, \tilde{\mathrm{m}}, \mathrm{k}}^{\mathrm{u}}+\mathrm{u} \sum_{\delta=2}^{\infty} \mathrm{b}_{\delta} \mu_{\mathrm{r}: \mathrm{n}, \tilde{\mathrm{m}}, \mathrm{k}}^{\mathrm{u}+-1}\right), \text { under } \mathrm{A}, \\
\frac{\mathrm{p}}{\mathrm{p} \theta \gamma_{\mathrm{r}}-\mathrm{u}}\left(\theta \gamma_{\mathrm{r}} \mu_{\mathrm{r}-1: \mathrm{n}, \tilde{\mathrm{m}}, \mathrm{k}}^{\mathrm{u}}+\mathrm{u} \sum_{\delta=2}^{\infty} \mathrm{b}_{\delta}^{\mathrm{u}} \mu_{\mathrm{r}: \mathrm{n}, \tilde{\mathrm{m}}, \mathrm{k}}^{\mathrm{u}+\delta-1}\right), \text { under B. }
\end{array}\right.
$$

Theorem 4. For $\mathrm{n}=1,2,3, \ldots, 1 \leq \mathrm{r}<\mathrm{s} \leq \mathrm{n}$ and $\mathrm{k} \geq 1$

$$
M_{r, s: n, \tilde{m}, k}\left(t_{1}, t_{2}\right)-M_{r, s-1: n, \tilde{m}, k}\left(t_{1}, t_{2}\right)
$$




$$
=\left\{\begin{array}{l}
\frac{\mathrm{t}_{2}}{\theta \gamma_{\mathrm{s}}}\left(\mathrm{M}_{\mathrm{r}, \mathrm{s}: \mathrm{n}, \tilde{\mathrm{m}}, \mathrm{k}}^{0,1}\left(\mathrm{t}_{1}, \mathrm{t}_{2}\right)+\sum_{\delta=2}^{\infty} \mathrm{b}_{\delta} \mathrm{M}_{\mathrm{r}, \mathrm{s}: \mathrm{n}, \tilde{\mathrm{m}}, \mathrm{k}}^{0, \mathrm{t}}\left(\mathrm{t}_{1}, \mathrm{t}_{2}\right)\right), \text { under } A, \\
\frac{\mathrm{t}_{2}}{\theta \gamma_{\mathrm{s}}}\left(\frac{\mathrm{M}_{\mathrm{r}, \mathrm{s}: \mathrm{n}, \tilde{\mathrm{m}}, \mathrm{k}}^{0,1}\left(\mathrm{t}_{1}, \mathrm{t}_{2}\right)}{\mathrm{p}}+\sum_{\delta=2}^{\infty} \mathrm{b}_{\delta}^{\prime} \mathrm{M}_{\mathrm{r}, \mathrm{s}: \mathrm{n}, \tilde{\mathrm{m}}, \mathrm{k}}^{0, \delta}\left(\mathrm{t}_{1}, \mathrm{t}_{2}\right)\right), \text { under } \mathrm{B} .
\end{array}\right.
$$

Proof. From (12), the joint moment generating function of $X(r, n, \tilde{m}, k)$ and $X(s, n, \tilde{m}, k)$ is given by

$$
\begin{aligned}
M_{r, s: n, \tilde{m}, k}\left(t_{1}, t_{2}\right)=c_{s-1} \int_{\alpha}^{\infty} \int_{x}^{\infty} e^{t_{1} x+t_{2} y} & {\left[\sum_{i=1}^{r} a_{i}(r)(\bar{F}(x))^{\gamma_{i}}\right] \frac{f(x)}{\bar{F}(x)} } \\
& \times\left[\sum_{i=r+1}^{s} a_{i}^{r}(s)\left(\frac{\bar{F}(y)}{\bar{F}(x)}\right)^{\gamma_{i}}\right] \frac{f(y)}{\bar{F}(y)} d y d x .
\end{aligned}
$$

Substituting $\omega(\mathrm{x}, \mathrm{y})=\mathrm{e}^{\mathrm{t}_{1} \mathrm{x}+\mathrm{t}_{2} \mathrm{y}}$ in (16) and using (5) for case A, we have

$$
\begin{array}{r}
M_{r, s: n, \tilde{m}, k}\left(t_{1}, t_{2}\right)-M_{r, s-1: n, \tilde{m}, k}\left(t_{1}, t_{2}\right)=\frac{t_{2} c_{s-1}}{\theta \gamma_{s}} \int_{\alpha}^{\infty} \int_{x}^{\infty} e^{t_{1} x+t_{2} y}\left[\sum_{i=1}^{r} a_{i}(r)(\bar{F}(x))^{\gamma_{i}}\right] \frac{f(x)}{\bar{F}(x)} \\
\times\left[\sum_{i=r+1}^{s} a_{i}^{r}(s)\left(\frac{\bar{F}(y)}{\bar{F}(x)}\right)^{\gamma_{i}}\right]\left[x+\sum_{\delta=2}^{\infty} b_{\delta} x^{\delta}\right] \frac{f(y)}{\bar{F}(y)} d y d x .
\end{array}
$$

On further simplification, (32) leads to the recurrence relation as stated in (30) for case A.

By repeating similar steps, the case B of (30) can easily be established.

Remark 4. After differentiating both sides of (30), with respect to $t_{1}$, $u$ times and with respect to $t_{2}, v$ times and putting $t_{1}=t_{2}=0$, we shall derive the recurrence relation for the product moments as

$$
\mu_{\mathrm{r}, \mathrm{s}: \mathrm{n}, \tilde{\mathrm{m}}, \mathrm{k}}^{\mathrm{u}, \mathrm{s}}=\left\{\begin{array}{l}
\frac{1}{\theta \gamma_{\mathrm{s}}-\mathrm{v}}\left(\theta \gamma_{\mathrm{s}} \mu_{\mathrm{r}, \mathrm{s}-1: \mathrm{n}, \tilde{\mathrm{m}}, \mathrm{k}}^{\mathrm{u}, \mathrm{v}}+\mathrm{v} \sum_{\delta=2}^{\infty} \mathrm{b}_{\delta} \mu_{\mathrm{r}, \mathrm{s}: \mathrm{n}, \tilde{\mathrm{m}}, \mathrm{k}}^{\mathrm{u}, \delta-1}\right), \text { under A, } \\
\frac{\mathrm{p}}{\mathrm{p} \theta \gamma_{\mathrm{s}}-\mathrm{v}}\left(\theta \gamma_{\mathrm{s}} \mu_{\mathrm{r}, \mathrm{s}-1: \mathrm{n}, \tilde{\mathrm{m}}, \mathrm{k}}^{\mathrm{u}, \mathrm{v}}+\mathrm{v} \sum_{\delta=2}^{\infty} \mathrm{b}_{\delta}^{\prime} \mu_{\mathrm{r}, \mathrm{s}: \mathrm{n}, \tilde{\mathrm{m}}, \mathrm{k}}^{\mathrm{u}, \mathrm{v}+\delta-1}\right), \text { under B. }
\end{array}\right.
$$

\section{Illustrated Examples}

In this section, we consider different forms of function $\Psi(\mathrm{x})$, which satisfy the desired restrictions $\Psi(0)=0, \Psi(\infty)=\infty$ and $\Psi_{\mathrm{n}}(0)<\infty, \mathrm{n}=1,2, \ldots$.

(a). Consider $\Psi(\mathrm{x})=\mathrm{x}$. In this case,

$$
\Psi_{1}(0) \neq 0 \text { and } \frac{\Psi(x)}{\Psi_{1}(x)}=x \text {. After comparing it with (3), we have } b_{\delta}=0, \delta=2,3, \ldots
$$


The pdf given in (1) becomes $\mathrm{f}(\mathrm{x})=\frac{\theta \alpha^{\theta}}{\mathrm{x}^{\theta+1}}, \quad \alpha \leq \mathrm{x}<\infty$. The recurrence relations in this case are obtained by putting $\mathrm{b}_{\delta}=0, \delta=2,3, \ldots$ in Remarks $1-4$ (case A) as follows:

$\mu_{\mathrm{r}: \mathrm{n}, \mathrm{m}, \mathrm{k}}^{\mathrm{u}}=\frac{\theta \gamma_{\mathrm{r}}}{\theta \gamma_{\mathrm{r}}-\mathrm{u}} \mu_{\mathrm{r}-1: \mathrm{n}, \mathrm{m}, \mathrm{k}}^{\mathrm{u}}$

(ii) $\quad \mu_{\mathrm{r}, \mathrm{s}: \mathrm{n}, \mathrm{m}, \mathrm{k}}^{\mathrm{u}, \mathrm{s}}=\frac{\theta \gamma_{\mathrm{s}}}{\theta \gamma_{\mathrm{s}}-\mathrm{v}} \mu_{\mathrm{r}, \mathrm{s}-1: \mathrm{n}, \mathrm{m}, \mathrm{k}}^{\mathrm{u}, \mathrm{v}}$

(iii) $\quad \mu_{\mathrm{r}: \mathrm{n}, \tilde{\mathrm{m}}, \mathrm{k}}^{\mathrm{u}}=\frac{\theta \gamma_{\mathrm{r}}}{\theta \gamma_{\mathrm{r}}-\mathrm{u}} \mu_{\mathrm{r}-1 \mathrm{n}, \tilde{\mathrm{m}}, \mathrm{k}}^{\mathrm{u}}$

(iv) $\quad \mu_{\mathrm{r}, \mathrm{s}: \mathrm{n}, \tilde{\mathrm{m}}, \mathrm{k}}^{\mathrm{u}, \mathrm{v}}=\frac{\theta \gamma_{\mathrm{s}}}{\theta \gamma_{\mathrm{s}}-\mathrm{v}} \mu_{\mathrm{r}, \mathrm{s}-1: \mathrm{n}, \tilde{\mathrm{m}}, \mathrm{k}}^{\mathrm{u}, \mathrm{s}}$

The deductions mentioned above in (i) and (ii) are verified by Theorems 2.1 and 3.1 of Athar et al. (2012) for Pareto distribution with parameter $\theta$.

(b). Consider $\Psi(\mathrm{x})=\mathrm{x}^{\beta}$, where $\beta$ is some positive integer greater than 1 . In this case $\Psi(0)=0, \Psi_{1}(0)=0, \Psi_{2}(0)=0, \ldots, \Psi_{\beta-1}(0)=0$ and $\Psi_{\beta}(0) \neq 0$. This case falls in category B. The pdf given in (1) now becomes $f(x)=\frac{\beta \theta \alpha^{\beta \theta}}{x^{\beta \theta+1}}, \quad \alpha \leq x<\infty$. The recurrence relations in this case are deduced by putting $\mathrm{p}=\beta$ and $\mathrm{b}_{\delta}^{\prime}=0, \delta=2,3, \ldots$ in Remarks $1-4$ (case B) and the same are given below:

$$
\mu_{\mathrm{r}: \mathrm{n}, \mathrm{m}, \mathrm{k}}^{\mathrm{u}}=\frac{\beta \theta \gamma_{\mathrm{r}}}{\beta \theta \gamma_{\mathrm{r}}-\mathrm{u}} \mu_{\mathrm{r}-1: \mathrm{n}, \mathrm{m}, \mathrm{k}}^{\mathrm{u}}
$$

(ii)

$$
\mu_{\mathrm{r}, \mathrm{s}: \mathrm{n}, \mathrm{m}, \mathrm{k}}^{\mathrm{u}, \mathrm{k}}=\frac{\beta \theta \gamma_{\mathrm{s}}}{\beta \theta \gamma_{\mathrm{s}}-\mathrm{v}} \mu_{\mathrm{r}, \mathrm{s}-1: \mathrm{n}, \mathrm{m}, \mathrm{k}}^{\mathrm{u}, \mathrm{s}}
$$

(iii)

$$
\mu_{\mathrm{r}: \mathrm{n}, \tilde{\mathrm{m}}, \mathrm{k}}^{\mathrm{u}}=\frac{\beta \theta \gamma_{\mathrm{r}}}{\beta \theta \gamma_{\mathrm{r}}-\mathrm{u}} \mu_{\mathrm{r}-1: \mathrm{n}, \tilde{\mathrm{m}}, \mathrm{k}}^{\mathrm{u}}
$$

(iv)

$$
\mu_{\mathrm{r}, \mathrm{s}: \mathrm{n}, \tilde{\mathrm{m}}, \mathrm{k}}^{\mathrm{u}, \mathrm{s}}=\frac{\beta \theta \gamma_{\mathrm{s}}}{\beta \theta \gamma_{\mathrm{s}}-\mathrm{v}} \mu_{\mathrm{r}, \mathrm{s}-1: \mathrm{n}, \tilde{\mathrm{m}}, \mathrm{k}}^{\mathrm{u}, \mathrm{s}}
$$

The results given above in (i) and (ii) are in agreement with the results of Athar et al. (2012) for Pareto distribution with parameter $\beta \theta$.

(c). Consider $\Psi(\mathrm{x})=\mathrm{e}^{\mathrm{x}}-1$. In this case $\Psi(0)=0$ and $\Psi_{1}(0) \neq 0$.

$$
\text { Now, } \frac{\Psi(\mathrm{x})}{\Psi_{1}(\mathrm{x})}=\frac{\mathrm{e}^{\mathrm{x}}-1}{\mathrm{e}^{\mathrm{x}}}
$$




$$
=1-\mathrm{e}^{-\mathrm{x}}=\mathrm{x}+\sum_{\delta=2}^{\infty} \mathrm{b}_{\delta} \mathrm{x}^{\delta}
$$

where $b_{\delta}=\frac{(-1)^{\delta-1}}{\delta !}$

The pdf in (1) now becomes $\mathrm{f}(\mathrm{x})=\frac{\theta \mathrm{e}^{\mathrm{x}}\left(\mathrm{e}^{\alpha}-1\right)^{\theta}}{\left(\mathrm{e}^{\mathrm{x}}-1\right)^{\theta+1}}, \alpha \leq \mathrm{x}<\infty$ and the recurrence relations in this case are obtained by putting $\mathrm{b}_{\delta}=\frac{(-1)^{\delta-1}}{\delta !}, \delta=2,3, \ldots$ in Remarks $1-4$ (case A) and are given below:

$$
\mu_{\mathrm{r}: \mathrm{n}, \mathrm{m}, \mathrm{k}}^{\mathrm{u}}=\frac{1}{\theta \gamma_{\mathrm{r}}-\mathrm{u}}\left(\theta \gamma_{\mathrm{r}} \mu_{\mathrm{r}-\mathrm{l}: \mathrm{n}, \mathrm{m}, \mathrm{k}}^{\mathrm{u}}+\mathrm{u} \sum_{\delta=2}^{\infty}\left(\frac{(-1)^{\delta-1}}{\delta !}\right) \mu_{\mathrm{r}: \mathrm{n}, \mathrm{m}, \mathrm{k}}^{\mathrm{u}+\delta-1}\right)
$$

$$
\mu_{\mathrm{r}, \mathrm{s}: \mathrm{n}, \mathrm{m}, \mathrm{k}}^{\mathrm{u}, \mathrm{v}}=\frac{1}{\theta \gamma_{\mathrm{s}}-\mathrm{v}}\left(\theta \gamma_{\mathrm{s}} \mu_{\mathrm{r}, \mathrm{s}-1: \mathrm{n}, \mathrm{m}, \mathrm{k}}^{\mathrm{u}, \mathrm{v}}+\mathrm{v} \sum_{\delta=2}^{\infty}\left(\frac{(-1)^{\delta-1}}{\delta !}\right) \mu_{\mathrm{r}, \mathrm{s}: \mathrm{n}, \mathrm{m}, \mathrm{k}}^{\mathrm{u}, \delta-1}\right)
$$

$$
\mu_{\mathrm{r}: \mathrm{n}, \tilde{\mathrm{m}}, \mathrm{k}}^{\mathrm{u}}=\frac{1}{\theta \gamma_{\mathrm{r}}-\mathrm{u}}\left(\theta \gamma_{\mathrm{r}} \mu_{\mathrm{r}-1: \mathrm{n}, \tilde{\mathrm{m}}, \mathrm{k}}^{\mathrm{u}}+\mathrm{u} \sum_{\delta=2}^{\infty}\left(\frac{(-1)^{\delta-1}}{\delta !}\right) \mu_{\mathrm{r}: \mathrm{n}, \tilde{\mathrm{m}, \mathrm{k}}}^{\mathrm{u}+\delta-1}\right)
$$

$$
\mu_{\mathrm{r}, \mathrm{s}: \mathrm{n}, \tilde{\mathrm{m}}, \mathrm{k}}^{\mathrm{u}, \mathrm{v}}=\frac{1}{\theta \gamma_{\mathrm{s}}-\mathrm{v}}\left(\theta \gamma_{\mathrm{s}} \mu_{\mathrm{r}, \mathrm{s}-1: \mathrm{n}, \tilde{\mathrm{m}}, \mathrm{k}}^{\mathrm{u}, \mathrm{v}}+\mathrm{v} \sum_{\delta=2}^{\infty}\left(\frac{(-1)^{\delta-1}}{\delta !}\right) \mu_{\mathrm{r}, \mathrm{s}: \mathrm{n}, \tilde{\mathrm{m}}, \mathrm{k}}^{\mathrm{u}+\delta-1}\right) \text {. }
$$

(d). Consider $\Psi(\mathrm{x})=\mathrm{e}^{\mathrm{x}}-\mathrm{x}-1$. We have, in this case, $\Psi(0)=0, \Psi_{1}(0)=0$ and $\Psi_{2}(0) \neq 0$.

$$
\text { Now, } \begin{aligned}
\frac{\psi(\mathrm{x})}{\psi_{1}(\mathrm{x})} & =\frac{\mathrm{e}^{\mathrm{x}}-\mathrm{x}-1}{\mathrm{e}^{\mathrm{x}}-1} \\
& =\sum_{\delta=1}^{\infty} \mathrm{b}_{\delta}^{\prime} \mathrm{x}^{\delta},
\end{aligned}
$$

where $\mathrm{e}^{\mathrm{x}}-\mathrm{x}-1=\left(\mathrm{e}^{\mathrm{x}}-1\right)\left(\sum_{\delta=1}^{\infty} \mathrm{b}_{\delta}^{\prime} \mathrm{x}^{\delta}\right)$

On comparing coefficients of like powers of $x$, we have $b_{1}^{\prime}=\frac{1}{2}, b_{2}^{\prime}=-\frac{1}{12}, b_{3}^{\prime}=0$ and, in general,

$$
(n+1)_{1} b_{1}^{\prime}+(n+1)_{2} b_{2}^{\prime}+\ldots+(n+1)_{n} b_{n}^{\prime}=1,
$$

where $\mathrm{n}=1,2, \ldots$ and $\mathrm{b}_{\delta}^{\prime}=0, \delta=0,-1,-2, \ldots$.

The pdf of the new class of Pareto distributions (1) for case B, with $p=2$, becomes

$$
f(x)=\frac{\theta\left(e^{x}-1\right)\left(e^{\alpha}-\alpha-1\right)^{\theta}}{\left(e^{x}-x-1\right)^{\theta+1}}, \alpha \leq x<\infty,
$$

and the recurrence relations in this case are obtained from Remarks $1-4$ (case B) as follows: 
(i)

$$
\begin{aligned}
& \mu_{\mathrm{r}: \mathrm{n}, \mathrm{m}, \mathrm{k}}^{\mathrm{u}}=\frac{2}{2 \theta \gamma_{\mathrm{r}}-\mathrm{u}}\left(\theta \gamma_{\mathrm{r}} \mu_{\mathrm{r}-\mathrm{l}: \mathrm{n}, \mathrm{m}, \mathrm{k}}^{\mathrm{u}}+\mathrm{u} \sum_{\delta=2}^{\infty} \mathrm{b}_{\delta}^{\prime} \mu_{\mathrm{r}: \mathrm{n}, \mathrm{m}, \mathrm{k}}^{\mathrm{u}+\mathrm{k}-1}\right) \\
& \mu_{\mathrm{r}, \mathrm{s}: \mathrm{n}, \mathrm{m}, \mathrm{k}}^{\mathrm{u}, \mathrm{v}}=\frac{2}{2 \theta \gamma_{\mathrm{s}}-\mathrm{v}}\left(\theta \gamma_{\mathrm{s}} \mu_{\mathrm{r}, \mathrm{s}-1: \mathrm{n}, \mathrm{m}, \mathrm{k}}^{\mathrm{u}, \mathrm{v}}+\mathrm{v} \sum_{\delta=2}^{\infty} \mathrm{b}_{\delta}^{\prime} \mu_{\mathrm{r}, \mathrm{s}: \mathrm{n}, \mathrm{m}, \mathrm{k}}^{\mathrm{u}, \mathrm{k}+\delta-1}\right) \\
& \mu_{\mathrm{r}: \mathrm{n}, \tilde{\mathrm{m}}, \mathrm{k}}^{\mathrm{u}}=\frac{2}{2 \theta \gamma_{\mathrm{r}}-\mathrm{u}}\left(\theta \gamma_{\mathrm{r}} \mu_{\mathrm{r}-1: \mathrm{n}, \tilde{\mathrm{m}, \mathrm{k}}}^{\mathrm{u}}+\mathrm{u} \sum_{\delta=2}^{\infty} \mathrm{b}_{\delta}^{\prime} \mu_{\mathrm{r}: \mathrm{n}, \tilde{\mathrm{m}, \mathrm{k}}}^{\mathrm{u}+\delta-1}\right) \\
& \mu_{\mathrm{r}, \mathrm{s}: \mathrm{n}, \tilde{\mathrm{m}}, \mathrm{k}}^{\mathrm{u}, \mathrm{v}}=\frac{2}{2 \theta \gamma_{\mathrm{s}}-\mathrm{v}}\left(\theta \gamma_{\mathrm{s}} \mu_{\mathrm{r}, \mathrm{s}-1: \mathrm{n}, \tilde{\mathrm{m}}, \mathrm{k}}^{\mathrm{u}, \mathrm{v}}+\mathrm{v} \sum_{\delta=2}^{\infty} \mathrm{b}_{\delta}^{\prime} \mu_{\mathrm{r}, \mathrm{s}: \mathrm{n}, \tilde{\mathrm{m}}, \mathrm{k}}^{\mathrm{u}, \mathrm{v}+\delta-1}\right) \text {. }
\end{aligned}
$$

\section{Conclusion}

In the study presented above, we demonstrate the recurrence relations for marginal and joint moment generating functions of GOS from a new class of Pareto distributions. These results generalize the corresponding results of Athar et al. (2012) for Pareto distribution.

\section{Acknowledgements}

Authors are grateful to Professor M. Ahsanullah, Editor - in - Chief, JSTA and the learned referees who spent their valuable time to review this manuscript.

\section{References}

[1] H. Athar and H. M. Islam, Recurrence relations between single and product moments of generalized order statistics from a general class of distributions, Metron, LXII (2004), 327 - 337.

[2] H. Athar, S. K. Khwaja and Nayabuddin, Expectation identities of Pareto distribution based on generalized order statistics. American Journal of Applied Mathematics and Mathematical Sciences, 1(2012), 23-29.

[3] N. Balakrishnan and P. C. Joshi, Product moments of order statistics from doubly truncated exponential distribution. Naval Res. Logist. Quart., 31(1984), 27-31.

[4] N. Balakrishnan and H. J. Malik, Order statistics from linear exponential distribution, Part I : Increasing hazard rate case. Commun. Statist. - Theor. Meth., 15(1986), 179-203.

[5] P. C. Joshi, Recurrence relations between moments of order statistics from exponential and truncated exponential distributions Sankhya, Ser. B, 39(1978), 362-371.

[6] P. C. Joshi, A note on mixed moments of order statistics from exponential and truncated exponential distributions. J. Statist. Plann. Inf., 6(1982), 13-16.

[7] U. Kamps, A concept of generalized order statistics, (B. G. Teubner, Stuttgart,1995a).

[8] U. Kamps, A concept of generalized order statistics, J. Statist. Plann. Inf., 48(1995b), 1-23.

[9] M. M. Mohie El-Din, M. A. W. Mahmoud, S. E. Abu-Youssef and K. S. Sultan, Order statistics from the doubly truncated linear exponential distribution and its characterizations. Commun. Statist.- Simul. Comput., 26(1997), 281-290.

[10] K. Nain, Recurrence relations for single and product moments of $\mathrm{k}^{\text {th }}$ record values from generalized Weibull distribution and a characterization. International Mathematical Forum, 5, No. 33(2010a), 1645 - 1652.

[11] K. Nain, Recurrence relations for single and product moments of ordinary order statistics from $\mathrm{p}^{\text {th }}$ order exponential distribution. International Mathematical Forum, 5, No. 34(2010b), 1653 - 1662.

[12] P. Pawlas and D. Szynal, Recurrence relations for single and product moments of generalized order statistics from Pareto, Generalized Pareto and Burr distributions. Commun. Statist. - Theor. Meth., 30(2001), 739-746.

[13] J. Saran and K. Nain, Recurrence relations for single and product moments of generalized order statistics from doubly truncated $\mathrm{p}^{\text {th }}$ order exponential distribution. Journal of the Kerala Statistical Association, 23(2012a), 01-16.

[14] J. Saran and K. Nain, Relationships for moments of kth record values from doubly truncated pth order exponential and generalized Weibull distributions. ProbStat Forum, 05(2012b), 142-149.

[15] J. Saran and K. Nain, Recurrence relations for single and product moments of $\mathrm{k}^{\text {th }}$ record values from $\mathrm{p}^{\text {th }}$ order exponential distribution and a characterization. Jahangirnagar University Journal of Statistical Studies, 31(2012c), 35-41.

[16] J. Saran and A. Pandey, Recurrence relations for single and product moments of generalized order statistics from linear 
exponential distribution. Journal of Applied Statistical Science, 13(2004), 323-333.

[17] J. Saran and A. Pandey, Recurrence relations for single and product moments of generalized order statistics from linear exponential and Burr distributions. Journal of Statistical Theory and Applications, 8, No. 3(2009), 383-391.

[18] J. Saran and A. Pandey, Recurrence relations for marginal and joint moment generating functions of dual generalized order statistics from inverse Weibull distribution. Journal of Statistical Studies, 30(2011), 65-72.

[19] J. Saran and N. Pushkarna, Moments of order statistics from doubly truncated linear exponential distribution. J. Korean Statist. Soc., 28(1999), 279-296. 